\title{
Design of a 20,000 pound variable stiffness actuator for structural vibration attenuation
}

\author{
John Leavitt, James E. Bobrow* and Faryar Jabbari \\ Department of Mechanical and Aerospace Engineering, University of California - Irvine, Irvine, CA 92697, USA
}

Received 6 July 2007

Revised 3 October 2007

\begin{abstract}
This paper describes the design of a novel actuator capable of protecting a full scale structure from severe load conditions. The design includes a cylinder filled with pressurized nitrogen and uses commercially available components. We demonstrate that the actuator behaves like a spring with an adjustable unstretched length, and that the effective spring stiffness can be changed easily by changing the initial cylinder pressure. In order to test the actuator on a full scale structure, an effective spring constant of approximately 10,000 pounds/inch was required over a two inch stroke. Because of the spring-like behavior, rather than damper-like behavior, the actuator does not transmit high forces to a vibrating structure like linear viscous dampers do when velocities are high. We analyze features of critical importance to the design of the actuator such as the cylinder dimensions, operating pressure, and valve selection. We then investigate the performance using a novel experimental apparatus that mimics the dynamics of a single story building, but has $1 / 400$ the weight.
\end{abstract}

\section{Introduction}

Actuators that are capable of protecting structures from severe load conditions are important for many applications. Typical applications include the reduction of building structural failures during earthquake and wind loading, and the isolation of human passengers and precision components in vehicles that move over rough terrain. An approach often used to achieve this protection is the incorporation of a passive damper as an integral part of the isolation system. In many instances this approach is warranted, but for some load conditions, dampers can make the problem worse. For instance, for low amplitude, high frequency excitations, linear viscous dampers amplify the force transmitted to a structure since the force from a damper is velocity-dependent rather than position-dependent, and velocity increases linearly with frequency. One method to reduce the force transmission is to use a power-law damper, where the output force can be approximated by $f=c v^{p}$ with $0<p<1[18,23]$. This power-law damper effectively limits the force transmitted to the structure when velocities are high.

As an alternative to power-law dampers, in this work we describe the mechanical and electrical design of an actuator capable of isolating a structure from high velocity loading or from motions with high frequency content. It uses a different method for extracting energy from the moving structure than viscous dampers do. The actuator is designed to have a 20,000 pound peak output force capability, so it has the potential to be used to protect full-scale structures. The specifications used here are derived from a set of actuators developed for full-scale testing on a research structure at the National Center for Research on Earthquake Engineering (NCREE) in Taipei, Taiwan, see [25]. We also describe a unique one-degree-of-freedom test apparatus used for performance testing of the hardware. The test apparatus behaves like a single story building, but it weighs much less, and allows us to conduct full-scale experiments without a full-scale building.

\footnotetext{
*Corresponding author. E-mail: jebobrow@uci.edu.
} 
The device developed in this work behaves like a controlled spring rather than a damper, so it does not transmit forces to a structure when velocities are high. The actuator is called a "semi-active" device, which is one that uses sensors and control logic to produce its output force, but requires little power consumption to do so and can never add energy to the vibrating structure. In our approach, the actuator behaves like a linear spring. However, at appropriate times, the effective unstretched length of the actuator is changed-or reset-to extract energy from the vibrating structure. The concept of stiffness elements that are reset to zero for extraction of energy was also used in $[14,3]$, in which a modal approach was used to study and design control laws for piezoelectric or piezoceramic devices with typical output levels considerably lower than our aim here. Analytical details of our approach have been presented in $[1,8,24]$, where it was shown that the settling time for transient response is faster for the variable stiffness approach presented here than for linear viscous dampers, and that the approach performs well for general structures with many modes of vibration. The design aspects developed in this work for a reset-able actuator capable of producing large output forces has not been published previously.

A comparison of other variable stiffness dampers has been conducted by Djajakesukma et al. [5] for buildings subject to different earthquake loadings. The testing of variable stiffness control algorithms on full-scale buildings has been conducted in $[10,13]$. The actuators used for these structures have high output force levels and can be expensive to make. A switched stiffness approach based on a velocity estimator has been conducted in [17]. There are only a few completely passive dampers with the high output force levels needed for structural vibration suppression commercially available today [22]. Semi-active control systems based on variable damping mechanisms have been the subject of much research over recent years. For instance, Jalili [9] analyzes and reviews various control system strategies using electro-rheological (ER) and magneto-rheological (MR) fluid dampers. Dyke et al. [6,16], have proposed a clipped optimal $\mathrm{H}_{2} / L Q G$ controller that outputs zero force when the device rod motion does not oppose the desired force. Ahmadian [19] has used an MR damper to investigate the so-called "sky-hook" control algorithm to providing passenger comfort in truck seat suspensions. One alternative to devices with variable damping or variable stiffness is a friction-based damper. These devices usually have two or more sliding surfaces in contact with another, which are pressed to together with some form of clamping mechanism [12,7]. Their advantages include zero fluid leakage, durable parts, and an insensitivity to temperature.

The results presented in this work are focused on the design of the actuator. In addition to the test results presented here, the desirable performance of the device was also evaluated in [11,25], via experiments and simulations, respectively. One of the key features of the proposed design is the reliance on standard, off-the-shelf components and materials to obtain high resisting forces. In this paper, we analyze features of importance to the design of the actuator so that one can choose the critical dimensions to achieve any stiffness and stroke. These include the cylinder dimensions, operating pressure, and valve selection.

\section{Resetting control for vibration attenuation}

In this section we review the concept of resetting control for a single degree of freedom system. More details, including the multi-story case, can be found in [8,24]. Figure 1(a) shows a model system, with $k_{0}$ representing the structural stiffness and $k_{1}$ representing the actuator stiffness. The spring $k_{1}$ and collar in Fig. 1(b) represents the conceptual behavior of the actuator shown on the right. If the collar is released, the mass moves with no force applied from the actuator. If the collar is fixed at some value $x_{s}$, the mass is subjected to a force from the actuator equal to $-k_{1}\left(x-x_{s}\right)$. The behavior of the collar is accomplished physically by opening and closing a valve that can block gas flow through a tube that connects the two sides of the cylinder, as shown in Fig. 1.

By opening an closing the valve at appropriate times, we can quickly remove energy from the vibrating system as follows. The equation of motion for the model system in Fig. 1 is

$$
m \ddot{x}+c \dot{x}+k_{0} x+k_{1}\left(x-x_{s}\right)=0,
$$

where $c$ is the damper constant, which includes structural damping, and linear viscous damping forces from the piston seals and the bearings. As mentioned previously, $k_{1}$ is the spring constant of the actuator, and $k_{0}$ is the spring constant of the structure. The variable $x$ is the current position of the mass, while $x_{s}$ is the position of the mass where resetting last occurred (the value of $x$ when the valve was last closed). With the valve closed at some point 

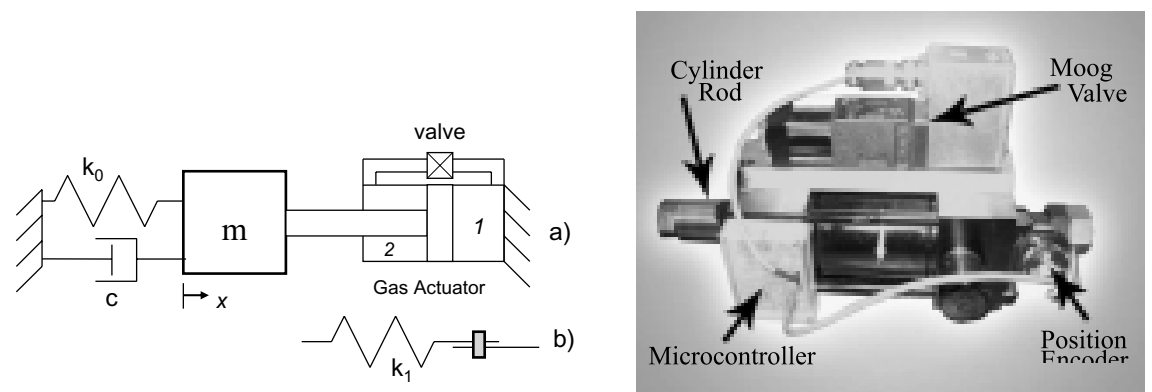

Fig. 1. Model mass-spring-damper system (left) and the hardware that implements resetting control (right).

$x_{s}$, the total energy of the system is

$$
E=U_{s}+U_{a}=\frac{1}{2}\left(m \dot{x}^{2}+k_{0} x^{2}\right)+\frac{1}{2} k_{1}\left(x-x_{s}\right)^{2}
$$

where $U_{s}$ is the kinetic plus potential energy of the structure, and $U_{a}$ is the energy stored in the actuator's spring. During the motion of the structure, energy flows back and forth between the structure and the actuator, and slowly dissipates through structural damping. It is desirable to transform the energy stored in the actuator into heat when it is at a maximum level and before it is transferred back to the structure. This occurs when $\dot{U}_{a}=0$, (or $\dot{U}_{a}$ changes sign), and $U_{a} \neq 0$. Since

$$
\dot{U}_{a}=k_{1} \dot{x}\left(x-x_{s}\right),
$$

a sign change can only occur when either $\left(x-x_{s}\right)$ changes sign or $\dot{x}$ changes sign. Because $U_{a} \neq 0$ implies that $x \neq x_{s}$, we conclude that the actuator's energy is at a maximum when $\dot{x}$ changes sign. It is at this moment when resetting event should occur. When resetting occurs, the valve is opened and the energy stored in the compressed gas is converted to heat. Conceptually, this heat release could be approximated by the energy that would be dissipated by a small mass and damper attached to $k_{1}$ in Fig. 1(b). Upon a reset event, the small mass-spring-damper system would vibrate and quickly dissipate the energy in $U_{a}$.

\section{Mechanical design of the resetable actuator}

In order to implement our concept for full scale structural systems, a device capable of producing high output force levels was needed that behaves like a resetable spring. To create the forces, we used a commercially available hydraulic cylinder as the actuator body, but filled it with compressed nitrogen, rather than oil. By using nitrogen, the effective spring "stiffness" can be adjusted by charging the cylinder to different pressures. In order to create the desired effect of "cutting a spring," or opening the collar, we used an on/off valve with a fast response time. In this section we develop an equation that relates the effective spring constant to the cylinder pressures and dimensions, and an equation that closely approximates the process of gas pressure equalization that occurs after the valve has been opened. This latter equation has not been presented previously, and is important because it can be used to choose the dimensions of the valve that controls the gas flow. Finally, we briefly discuss some of the design considerations encountered during the implementation of our control logic on a low-cost microcontroller.

\subsection{Spring-like behavior of the system}

Referring the actuator shown in Fig. 2, we develop an analytical expression for the effective "spring constant" that is obtained when the valve that connects the two sides of the cylinder is closed. We show that this spring constant is directly proportional to the initial pressure in the cylinder. Given that $V_{0}$ is the volume on either side of the piston when $x=0$, (we assume $x=0$ at the midstroke position of the piston), and that $A$ is the area of the piston, then the volumes on sides one and two of the piston, as marked in Fig. 2, are 


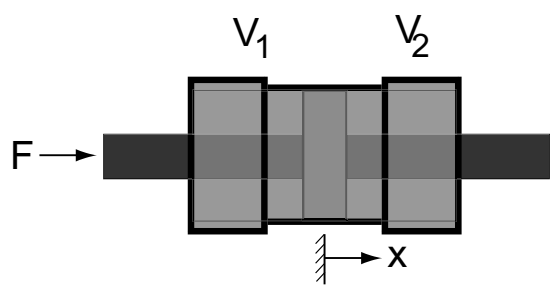

Fig. 2. Nitrogen-filled cylinder.

$$
\begin{aligned}
& V_{1}=V_{0}-A x \\
& V_{2}=V_{0}+A x .
\end{aligned}
$$

Assuming that we have a closed adiabatic system containing an ideal gas, the quantity [4]

$$
c=P_{1} V_{1}^{\gamma}=P_{2} V_{2}^{\gamma}
$$

is constant, where $P_{1}$ and $P_{2}$ are the pressures in volumes 1 and $2, \gamma$ is the ratio of specific heats for nitrogen, and $c=P_{0} V_{0}^{\gamma}$, given the known initial conditions $P_{0}$ and $V_{0}$. It follows that the force from the gas on the piston is

$$
F=\left(P_{2}-P_{1}\right) A=\left[\left(V_{0}+A x\right)^{-\gamma}-\left(V_{0}-A x\right)^{-\gamma}\right] A P_{0} V_{0}^{\gamma},
$$

as a function of $\mathrm{x}$. For motion of the piston near $x=0, F(x)$ can be approximated by

$$
F(x) \cong \frac{d F(0)}{d x} x=-\frac{2 A^{2} \gamma P_{0}}{V_{0}} x .
$$

Viewing the cylinder as a spring, from Eq. (2) the spring constant is

$$
k=\frac{2 A^{2} \gamma P_{0}}{V_{0}} .
$$

Note that $k$ is directly proportional to $P_{0}$, the pressure that the cylinder is initially charged to. Equation (3) is used to choose the cylinder dimensions (bore and stroke) and operating pressure $P_{0}$ in order to achieve a given stiffness $k$. This can be done for a given maximum cylinder operating pressure as defined by the manufacturer as follows. For a given maximum cylinder pressure $P_{\max }$, desired stiffness $k$, and maximum piston travel $x_{\max }$, we have $F=k x_{\max }=P_{\max } A$, which gives the area $A$, and hence the cylinder bore. Once the bore has been chosen, the stroke must be longer than $2 x_{\max }$ (the cylinder moves a total distance of $\pm x_{\max }$ ), and this choice defines $V_{0}$. Once $V_{0}$ is known, $P_{0}$ can be chosen to give the desired value of $k$. In our case, $x_{\text {max }}$ was 1.0 inches, $k$ was $10,000 \mathrm{lb} / \mathrm{inch}$, and $p_{\max }$ was 3000 psi. This led to the selection of a cylinder bore of 4.0 inches, a stroke of 3.0 inches, with an operating pressure $P_{0}$ of about 700 psi.

We tested the stiffness relationship at various initial cylinder pressures, while keeping the control valve closed. Oscillating the pendulum shown in the next section by hand, we recorded the rod position and the pressure on each side of the actuator as functions of time. Figure 3 shows data taken from the actuator when it was initially charge to a pressure of 285 psia, plotted on the left versus time. To test for linearity, the rod position has been scaled. Note that over the entire motion, the net force coincides directly with the scaled position, indicating that the linearized analysis is justified. This linear relationship was seen in all of the tests we performed. Next, to determine the spring constant of the cylinder, we plotted the net force versus the rod position, and fit a line to the data. The right-hand plot in Fig. 3 shows the best fit line for the data. The slope of the line is $4390 \mathrm{lbs} / \mathrm{in}$ and the value predicted by Eq. (3) was $4432 \mathrm{lbs} / \mathrm{in}$. This value is slightly higher than the measured spring constant-probably because it is difficult to measure the exact cylinder volume $V_{0}$. We repeated this process 5 different initial pressures, and for another cylinder, and found that the predicted value in Eq. (3) was always within $1 \%$ of the value that we measured experimentally. 

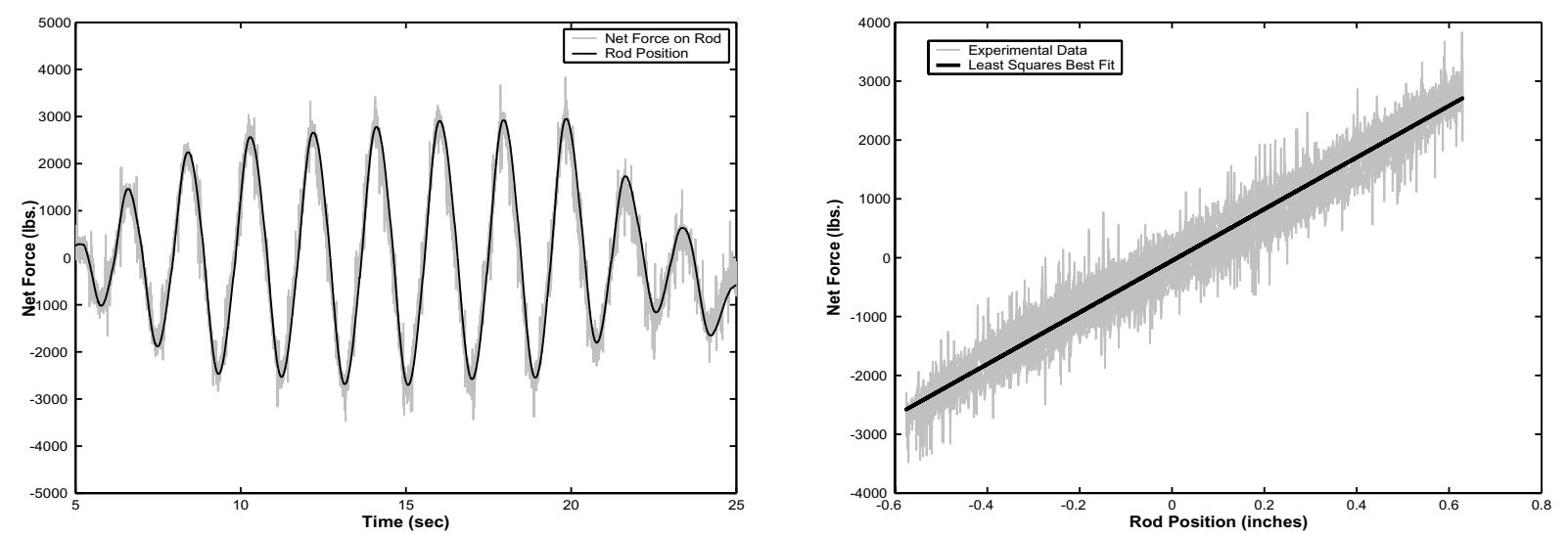

Fig. 3. Rod position (scaled) and force versus time (left) and rod force versus position (right). Both with the valve closed and an initial pressure of $P=285$ psia.

\subsection{Cylinder reset time}

Another important parameter besides the effective cylinder stiffness is the time that it takes to achieve a reset. For this, valve selection is a critical consideration. A fast reset time is desirable since this determines the range of frequencies for which the actuator can remove energy from the system. We looked at both the speed it takes to fully open the valve (the step response), as well as the amount of flow the valve allows once it is open. Valves that give high flow rates use larger moving components, move slower, require more power, and are more expensive, than smaller valves. Commercially available valves that are rated for the pressures needed for this work are designed for hydraulic systems, and data is not available for their gas flow characteristics. The analysis below allows us to estimate the response time of a given valve once the orifice has opened.

Consider a control volume $V$ at pressure $P$ and temperature $T$ with a gas flowing into it. Assuming that $\dot{V}=0$ and that the system is adiabatic, the energy equation [4] is

$$
\frac{d}{d t}\left(c_{p} T_{s} m\right)=\frac{d}{d t}\left(c_{v} T \rho V\right)
$$

where $c_{p} T_{s}$ is the specific energy of the incoming gas at temperature $T_{s}$, and $c_{v} T$ is the specific energy of the gas in volume $V$ with mass $\rho V$. Differentiating Eq. (4) with resect to time, and assuming that $T_{s}=T$, and $\dot{V} \approx 0$ (the cylinder motion is slow compared to the rate of change of pressure), we have $c_{p} \dot{m}=c_{v} \dot{\rho} V$. Then, using the time derivative of the ideal gas law $P V=\rho V R T$ to eliminate $\dot{\rho}$, we obtain the rate of change of pressure on each side of the piston:

$$
\dot{P}_{1}=\gamma \frac{\dot{m}_{1} R T}{V_{1}}
$$

and

$$
\dot{P}_{2}=\gamma \frac{\dot{m}_{2} R T}{V_{2}}
$$

where $\gamma=\left(\frac{c_{p}}{c_{v}}\right)$. Because all of the gas that flows out of one chamber flows into the other,

$$
\dot{m}_{1}=-\dot{m}_{2} \text {. }
$$

Although the flow of a gas through an orifice is complex, we make the following linear approximation [2]

$$
\dot{m}_{1} \approx c_{\text {flow }}\left(P_{2}-P_{1}\right) \text {, }
$$

for some constant $c_{\text {flow }}$. This constant is directly proportional to the valve orifice area. Substituting Eqs (8) and (7) into Eqs (5) and (6), gives 


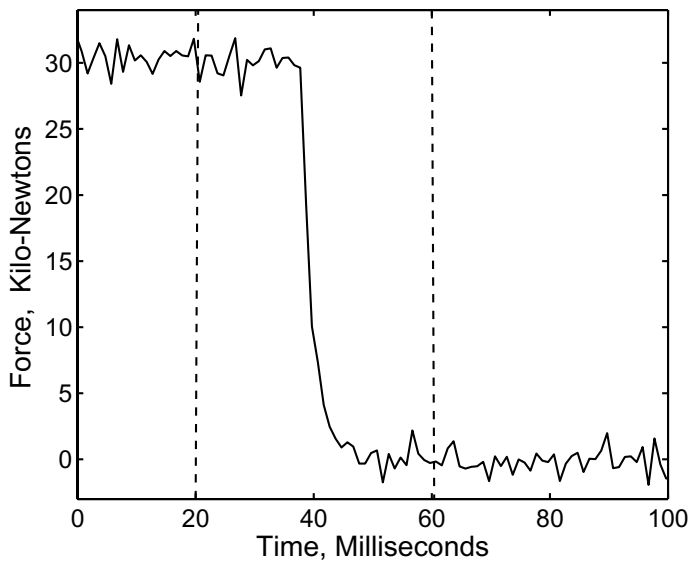

Fig. 4. The force reset event occurs in .040 seconds between the dotted vertical lines.

$$
\begin{aligned}
& \dot{P}_{1}=\frac{\gamma c_{\text {flow }} R T}{V_{1}}\left(P_{2}-P_{1}\right) \\
& \dot{P}_{2}=-\left(\frac{V_{1}}{V_{2}}\right) \dot{P}_{1} .
\end{aligned}
$$

Differentiating Eq. (9) again and substituting Eq. (10) into the result, gives

$$
\ddot{P}_{1}=-\dot{P}_{1} / \tau,
$$

where $\tau \equiv \frac{V_{1} V_{2}}{\left(V_{1}+V_{2}\right)\left(k c_{\text {flow }} R T\right)}$. The solution to Eq. (11) is

$$
P_{1}(t)=P_{1}(\infty)+\left(P_{1}(0)-P_{1}(\infty)\right) e^{-\frac{t}{\tau}}
$$

where $P_{1}(\infty)$ is the pressure at equalization, and we have assumed that the system is not moving $(\dot{V}=0 \Rightarrow$ $\tau=$ constant). In order to make the system respond quickly, the time constant $\tau$ can be made small by increasing the size of the valve orifice $c_{\text {flow }}$. Furthermore, $\tau$ achieves its minimum value at either end of the piston stroke, since either $V_{1}$ or $V_{2}$ is a minimum at these points. Physically, the net force changes quickly at these locations because small changes in mass flow have a large influence on the pressure when the chamber volume is small. The worst case, or slowest value for the response is at the midstroke position, where $\tau=\frac{V_{0}}{2 k c_{\text {flow }} R T}$ (note at midstroke $V_{0}=V_{1}=V_{2}$.) From this analysis, we conclude that near the ends of the piston travel, or for a large valve orifice and tubing, the pressures will equalize relatively quickly and produce the desired effect of a fast resetting spring.

We tested several commercially available valves, and found the Moog D633 proportional valve gave a fast overall cylinder reset time. This valve uses a single stage solenoid to drive the internal spool, and thus has a relatively fast step response. Figure 4 shows one representative result of tests we conducted to determine the response time of the actuator and valve combination. In this Figure, the net force on the piston (as measured with pressure transducers on each side) is plotted versus time. The vertical dotted lines indicate the duration that control signal was sent to the valve in order to hold it open. One can see from the plot that there is a delay before the force quickly drops. The initial delay of about .017 seconds is caused by the step response of the valve itself. The total time for the reset event is less than 0.040 seconds. This is consistent with previous results in [1] for a much smaller device which has a total reset time of approximately 0.020 seconds. We note that the time delay and flow constants are not often provided by the manufacturer, so it is difficult to predict accurately the results shown in Fig. 4 without testing.

\subsection{Microcontroller logic}

The control logic was implemented on a Rabbit [15] microcontroller, which has an on-board quadrature decoder and digital outputs. The cylinder rod position was measured by an optical encoder [21], and the valve was controlled 

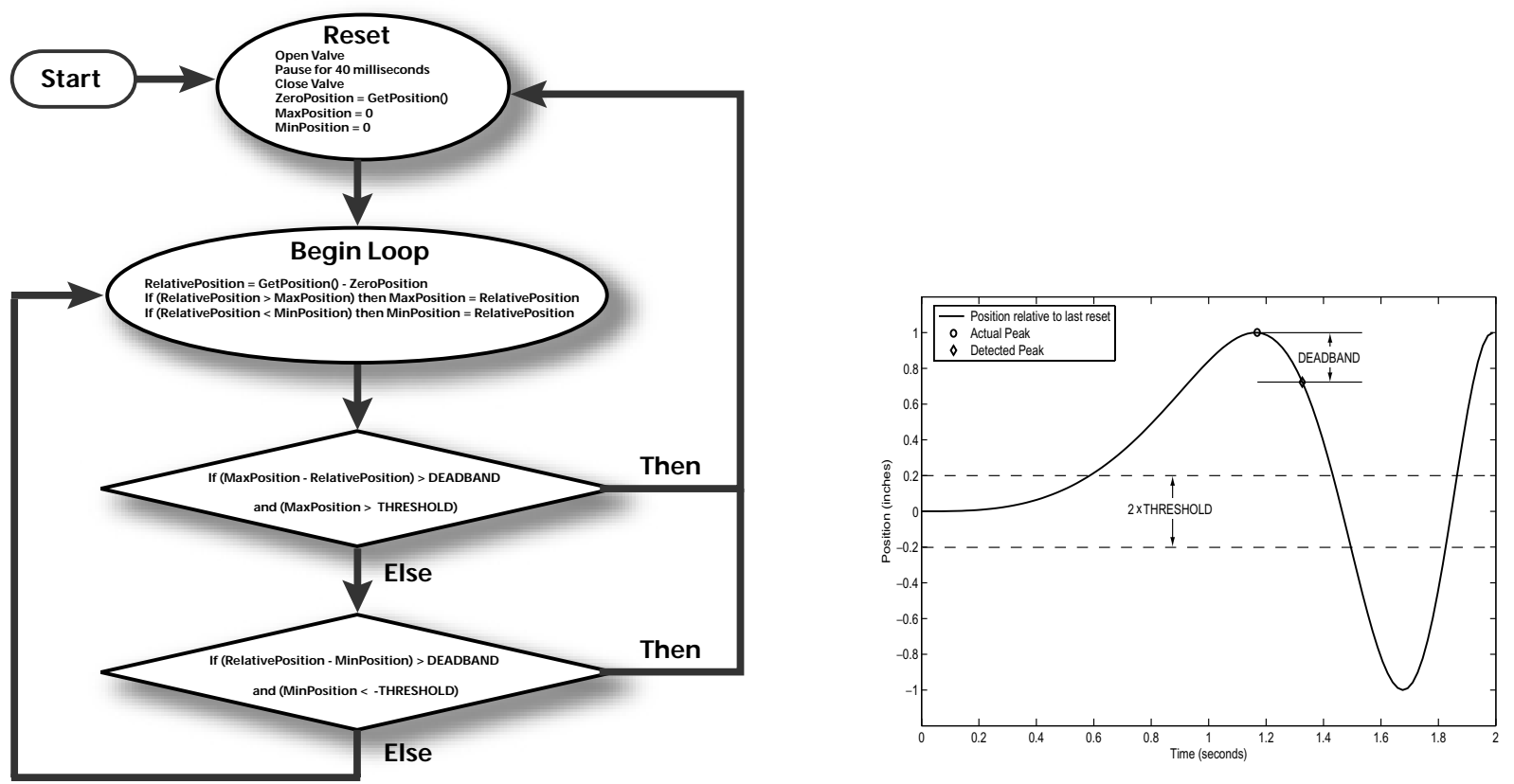

Fig. 5. Flow chart of resetting controller (left) and parameters affecting peak detection logic (right). For this actuator DEADBAND $=0.27 "$ and THRESHOLD $=0.2^{\prime \prime}$

with digital output signals. The control logic determines the times at which $\dot{x}=0$ by looking for the peaks or valleys in the piston displacement. At these times the actuator pressure is released by opening the Moog valve for 0.040 seconds and then closing it. In addition, we introduced a small deadband $\epsilon$ around the position signal such that the controller will not detect a peak and subsequently reset until the current position is a distance $\epsilon$ below the peak or above a valley. This feature also helps to eliminate the detection of peaks due to electrical noise or to high frequency, low amplitude vibrations in the structure.

The flowchart shown in Fig. 5, shows that only two parameters, DEADBAND $=\epsilon$ mentioned above, and THRESHOLD are used to define the peak detection logic implemented in the microcontroller. Both parameters eliminate the false peaks and unnecessary resets that result from measurement noise, as well as from high frequency/low amplitude vibrations in the building. The controller will not detect a high peak if the rod has not moved a positive distance THRESHOLD from last reset position. Likewise, a low peak is not detected if the rod has not a moved the same distance in the opposite direction from the last reset position. This feature assures that a minimum amount of energy is removed from the system each time the cylinder resets (recall that energy release is proportional to the square of $\delta x$.

We note that although our control algorithm finds peaks in the actuator position, as shown in Fig. 3, these peaks correspond exactly with the peaks in the force exerted on the piston by the gas when the valve is closed. Originally, we tried to base our control logic on the force measurements made with pressure sensors, but the signal noise created by the Moog valve was excessive. Figure 3 also shows the noise from the force measurements.

\section{A light-weight test apparatus that mimics large masses}

A test apparatus was constructed to investigate the vibration suppression capability of the actuator. The apparatus has an equation of motion similar to the one-degree-of freedom system shown in Fig. 1. We first considered constructing a system with a translating mass. However, because we needed an actuator stiffness of about 10,000 pounds/inch, and typical structural frequencies are a few Hertz, we needed a prohibitively large translating mass. For instance, for a natural frequency of about $1.5 \mathrm{Hertz}, \omega^{2}=k / m$ requires a mass that weighs about 50,000 pounds, 

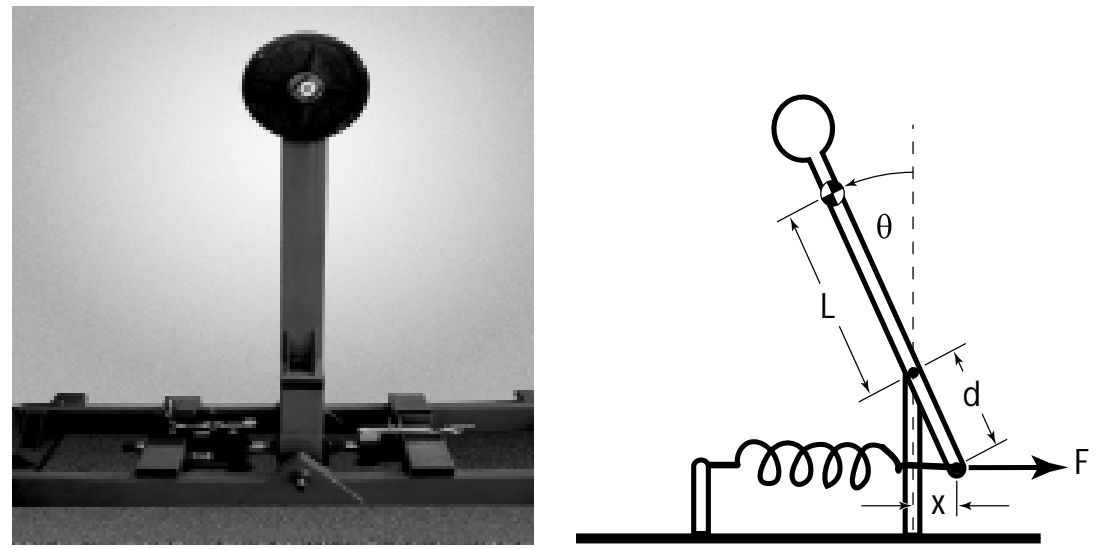

Fig. 6. Photo and schematic diagram of pendulum-like test apparatus. The unit is approximately 5 feet tall and the upper mass weighs approximately 100 pounds.

which is not feasible for a laboratory test stand. In order to circumvent this problem, we built the apparatus shown in Fig. 6.

The spring shown in the schematic, that represents the structure $k_{0}$ in Eq. (1), was implemented as another gasfilled cylinder. Because our actuator had a stroke of three inches, we constrained the apparatus to a corresponding range of motion of about $\pm 30^{\circ}$. Over this range of motion, the dynamics of the apparatus approximate those of the desired mass-spring-damper system. Around its pivot point, an inverted pendulum of mass $m$ has a moment of inertia $I=m L^{2}+I_{c}$, where $I_{c}$ is the moment of inertia about the center of mass of the pendulum. Assuming the spring remains approximately horizontal during the pendulum's motion, then the spring displacement is approximately equal to $x=d \sin (\theta)$. The torque generated by the spring about the pivot point is

$$
\tau_{s}=-(k x+c \dot{x}) d \cos \theta=-k d^{2} \sin \theta \cos \theta-c \dot{\theta} d^{2} \cos ^{2} \theta .
$$

The torque generated by gravity is $u_{g}=m g L \sin \theta$. And finally, assuming that the control cylinder remains approximately horizontal, its applied torque is $\tau_{c}=F d \cos \theta$. Summing these torques, we obtain the equation of motion

$$
I \ddot{\theta}+c \dot{\theta} d^{2} \cos ^{2} \theta+k d^{2} \sin \theta \cos \theta-m g L \sin \theta-F d \cos \theta=0 .
$$

Assuming that $\sin \theta \approx \theta$ and $\cos \theta \approx 1$, then

$$
I \ddot{\theta}+c d^{2} \dot{\theta}+\left(k d^{2}-m g L\right) \theta-F d=0
$$

and since $x=\theta d, \ddot{x}=\ddot{\theta} d$, we obtain the linearized differential equation of the pendulum in terms of $x$ :

$$
m_{b} \ddot{x}+c \dot{x}+k_{b} x=F
$$

where $m_{b}=\left(\frac{m L^{2}+I_{c}}{d^{2}}\right)$ and $k_{b}=\left(k-\frac{m g L}{d^{2}}\right)$. Notice that $m_{b}$ varies directly with $L^{2}$ and inversely with $d^{2}$. By making $L$ large and $d$ small, we are able to obtain systems with effective masses that are much larger than their actual mass $m$. For the test apparatus shown, we used $L / d=20$, with a pendulum weight of 100 pounds. This gave weight for $m_{b}$ of about 50,000 pounds as was desired.

We performed various experiments on the test hardware with the control logic turned on and off, and with different initial conditions. Figure 7 shows a typical result. An initial condition was created experimentally by using a 4 ton winch placed on the upper mass and cranking the winch to displace the mass. We used a quick-release mechanism attached to the winch cable in order to enable the system to move freely from this initial condition. The solid line shows a typical response with the control logic on, and the dashed line shows the response with the device turned off. The steady state error in the equilibrium position is due to dry friction in our test apparatus, including the cylinders and seals. Note that this improved damper-like behavior has been achieved with a damper that dissipates stored potential energy in short bursts. 


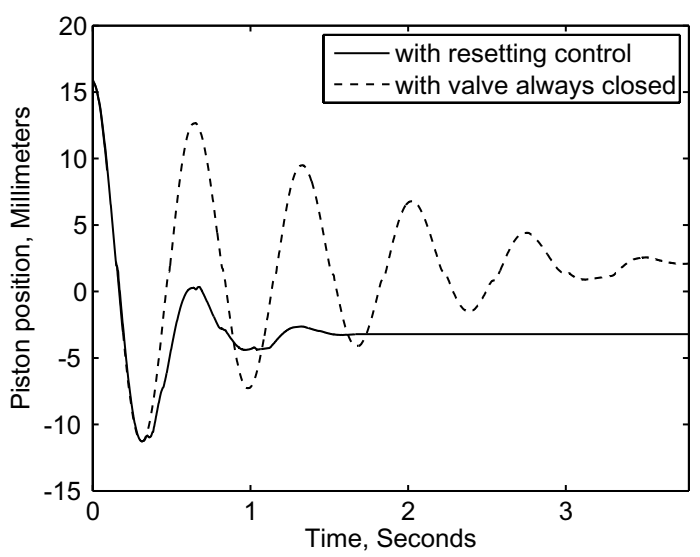

Fig. 7. Response of pendulum system with resetting control logic turned on (solid) and off (dotted). The offset shown is due to Coulomb friction.

\section{Conclusion}

The electromechanical design aspects of a new actuator capable of high output force levels has have been described. The gas-filled actuator can be constructed from reliable, commercially available components. We show with analysis and experiments that the actuator behaves like a spring with an adjustable unstretched length, and that the effective spring stiffness can be changed easily by changing the initial cylinder pressure. We develop an expression for the response time of the system, which can be used to determine the valve orifice diameter. We show that by opening the valve, the effective spring force quickly released, thereby converting stored energy into heat. We demonstrated the performance of the actuator on an novel experimental apparatus that mimics the dynamics of a single story building, but has $1 / 400$ the weight.

\section{Acknowledgement}

The authors would like to thank Professor Jann Yang, from the University of California, Irvine for his definition of the actuator specifications, and for the testing done at the National Center for Research on Earthquake Engineering (NCREE) in Taiwan. The results are reported in [25].

\section{References}

[1] J.E. Bobrow, F. Jabbari and K. Thai, An active truss element and control law for vibration suppression, Smart Materials Structures 4 (December 1995), 264-269.

[2] J.E. Bobrow and B. McDonell, Modeling, Identification, and Control of a Pneumatically Actuated, Force Controllable Robot, IEEE Transactions on Robotics and Automation 14(5) (October 1998), 732-742.

[3] L.R. Corr and W.W. Clark, A Novel Semi-Active Multi-Modal Vibration Control Law for a Piezoceramic Actuator, ASME Journal Of Vibration And Acoustics 125 (April 2003), 214-222.

[4] R.L. Daugherty, J.B. Franzini and E.J. Finnemore, Fluid Mechanics with Engineering Applications, McGraw-Hill Book Company, 1985.

[5] S.L. Djajakesukma, B. Samali and H. Nguyen, Study of a semi-active stiffness damper under various earthquake inputs, Earthquake Engineering and Structural Dynamics 31(10) (October 2002), 1757-1776.

[6] S.J. Dyke, S.J. Spencer Jr., M.K. Sain and J.D. Carlson, Seismic response reduction using magneto-rheological dampers, Proceddings 1996 IFAC 13th Triennial World Congress, San Francisco, 145-150.

[7] W.L. He, A.K. Agrawal and J.N. Yang, Novel semiactive friction controller for linear structures against earthquakes, Journal of Structural Engineering 129(7) (2003), 941-950.

[8] F. Jabbari and J.E. Bobrow, Vibration Suppression with a Resettable Device, Journal of Engineering Mechanics 128(9) (September, 2002), 916-914.

[9] N. Jalili, A Comparative Study and Analysis of Semi-Active Vibration-Control Systems, ASME Journal Of Vibration And Acoustics 124 (October 2002), 593-605. 
[10] T. Kobori and S. Kamagata, Dynamic intelligent buildings: Active seismic response control, Intelligent structures; monitoring and control, Y. K. Wen, ed., Elsevier Applied Science, New York, 1991, pp. 279-282.

[11] J.L. Leavitt, J.E. Bobrow, F. Jabbari and J.N. Yang, Application of a high-pressure gas semi-active resetable damper to the benchmark smart base-isolated buildings, Journal of Structural Control and Health Monitoring 13 (2006), 748-757.

[12] L.Y. Lu, Predictive control of seismic structures with semi-active friction dampers, Earthquake Engineering and Structural Dynamics 33(5) (2004), 647-668.

[13] N. Niwa, T. Kobori, M. Takahashi, H. Midorikawa, N. Kurata and T. Mizuno, Dynamic loading test and simulation analysis of full-scale semi-active hydraulic damper for structural control, Earthquake Engineering and Structural Dynamics 29(6) (2000), 789-812.

[14] J. Onoda, T. Endot, H. Tamaoki and N. Watanabe, Vibration Suppression by Variable-Stiffness Members, AIAA Journal 29(6) (1991) 0001-1452, 977-983.

[15] http://www.rabbitsemiconductor.com.

[16] J.C. Ramallo, E.A. Johnson, B.F. Spencer, Jr. and M.K. Sain, Semi-active building base isolation, Proceedings of the 1999 American Control Conference 1 (2-4 June 1999), 515-519.

[17] A. Ramaratnam and N. Jalili, A switched stiffness approach for structural vibration control: Theory and real-time implementation, Journal of Sound and Vibration 291(1-2) (Mar 21, 2006), 258-274.

[18] F. Rudinger, Tuned mass damper with nonlinear viscous damping, Journal of Sound and Vibration 300(Issues 3-5) (6 March 2007), 932-948.

[19] D. Simon and M. Ahmadian, Vehicle Evaluation of the Performance of Magneto Rheological Dampers for Heavy Truck Suspensions, ASME Journal Of Vibration And Acoustics 123 (July 2001), 365-375.

[20] N.D. Sims, D.J. Peel, R. Stanway, A.R. Johnson and W.A. Bullough, The Electrorheological long-stroke damper: a new modelling technique with experimental validation, Journal of Sound and Vibration 229(2) (Jan, 2000), 207-227.

[21] http://spaceagecontrol.com.

[22] D.P. Taylor and M.C. Constantinou, Testing procedures for high output fluid viscous dampers used in building and bridge structures to dissipate seismic energy, Shock and Vibration Digest 2(5) (1995), 373-381.

[23] G. Terenzi, Dynamics of SDOF systems with nonlinear viscous damping, Journal of Engineering Mechanics 125 (1999), $956-963$.

[24] J.N. Yang, J.-H. Kim and A.K. Agrawal, Resetting semiactive stiffness damper for seismic response control, Journal of Structural Engineering 126(12) (December 2000), 1427-1433.

[25] J.N. Yang, J. Bobrow, F. Jabbari, J. Leavitt, C.P. Cheng and P.Y. Lin, Full scale experimental verification of resetable semi-active stiffness dampers, Earthquake Engineering and Structural Damping 26 (2007), 1255-1273. 

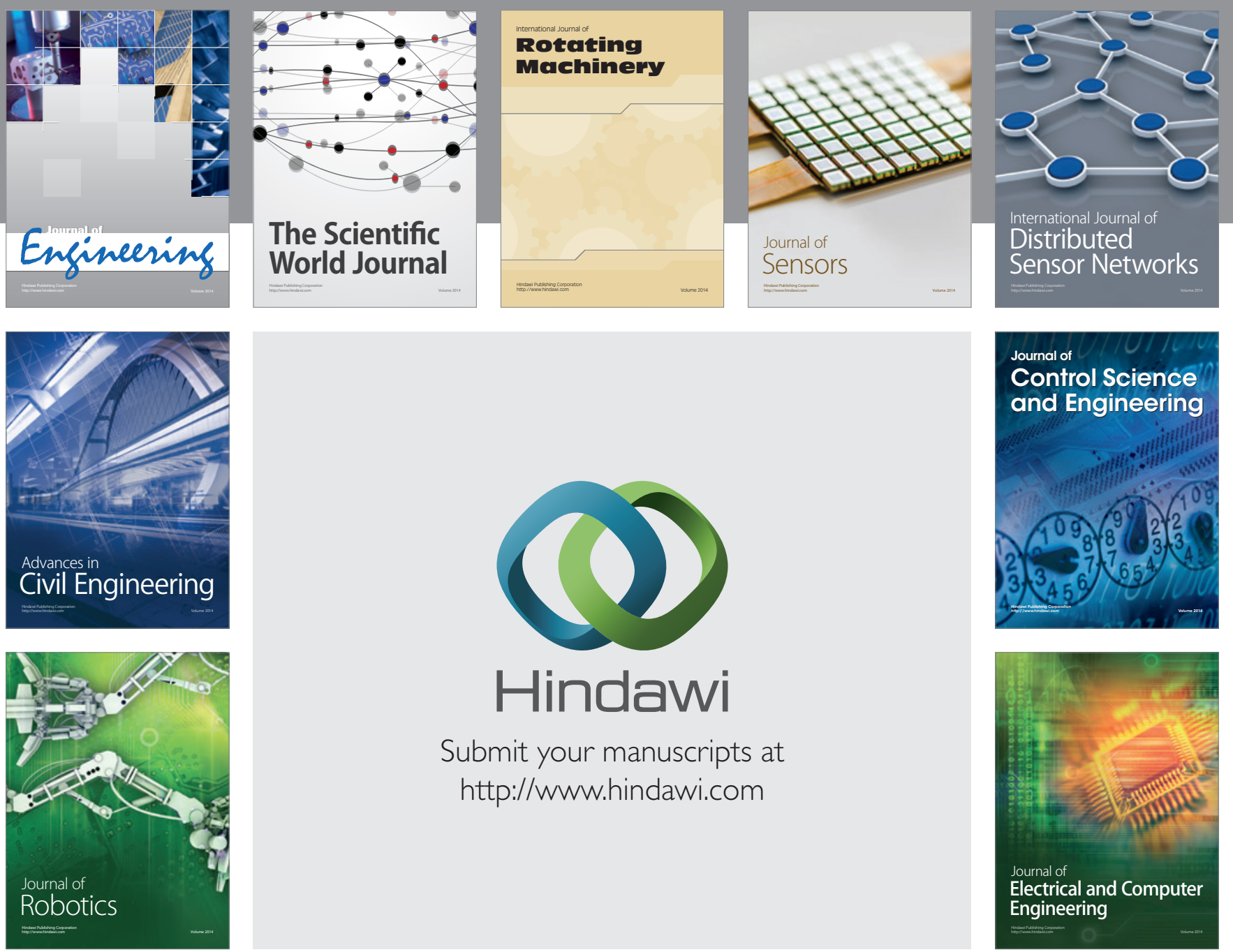

Submit your manuscripts at

http://www.hindawi.com
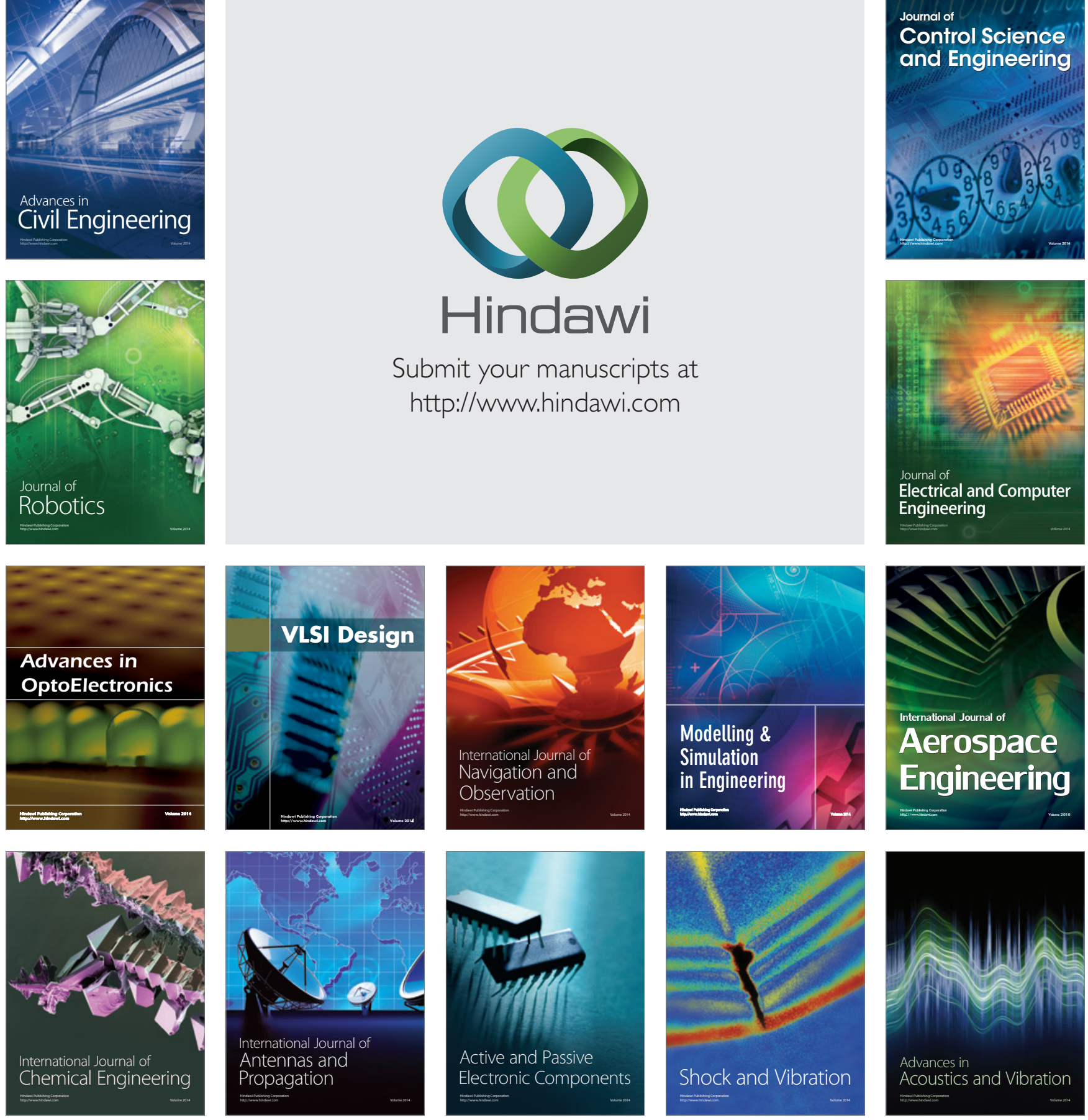